\title{
BIBLIOGRAPHY
}

1. R. R. Phelps, Extreme points in function algebras, Duke Math. J. 32 (1965), 267-277.

YaLe University

\section{ON THE OSCILLATION OF SUMS OF RANDOM VARIABLES}

\section{L. HANSON ${ }^{1}$ AND MELVIN KATZ ${ }^{2}$}

The object of this note is to prove the following theorem which may be interpreted as stating that sums of independent and identically distributed random variables do not oscillate too violently.

THEOREм. Let $\left\{X_{k}: k \geqq 1\right\}$ be a sequence of independent and identically distributed random variables and let $S_{n}=\sum_{k=1}^{n} X_{k}$. The series $\sum_{n=1}^{\infty}(-1)^{n} n^{-1} P\left(S_{n}>0\right)$ is convergent.

Proof. Let $l=P\left\{\bigcap_{\mathbf{k}=1}^{\infty}\left[S_{k}>0\right]\right\}$ and $l^{\prime}=P\left\{\bigcap_{\mathbf{k}=1}^{\infty}\left[S_{k} \leqq 0\right]\right\}$. It is known, [2], that for $|t|<1$

$$
\sum_{n=0}^{\infty} t^{n} P\left\{\bigcap_{k=1}^{n}\left[S_{k}>0\right]\right\}=\exp \left\{\sum_{k=1}^{\infty} t^{k} k^{-1} P\left[S_{k}>0\right]\right\} .
$$

If $l=0$ then $\sum_{n=0}^{\infty}(-1)^{n} P\left\{\bigcap_{k=1}^{n}\left[S_{k}>0\right]\right\}$ is convergent. It follows from (1), and the Littlewood Tauberian Theorem that

$$
\sum_{n=1}^{\infty}(-1)^{n} n^{-1} P\left(S_{n}>0\right)
$$

is convergent. Similarly, if $l^{\prime}=0$ then $\sum_{n=1}^{\infty}(-1)^{n} n^{-1} P\left(S_{n} \leqq 0\right)$ is convergent which implies $\sum_{n=1}^{\infty}(-1)^{n} n^{-1} P\left(S_{n}>0\right)$ is convergent.

For the case $l>0$ and $l^{\prime}>0$ we proceed differently. $\sum_{n=1}^{\infty}(-1)^{n} n^{-1}$ is convergent and thus it suffices to prove

$$
\sum_{n=1}^{\infty}\left|P\left(S_{n}>0\right)-P\left(S_{n+1}>0\right)\right|<\infty .
$$

However this last sum is bounded by $\sum_{n=1}^{\infty}\left\{P\left(S_{n}>0, S_{n+1} \leqq 0\right)\right.$ $\left.+P\left(S_{n} \leqq 0, S_{n+1}>0\right)\right\}$. Since $l>0$ and $l^{\prime}>0$ it follows by a standard

Received by the editors July 22, 1965.

1 Research supported by the Air Force Office of Scientific Research.

2 Supported in part by National Science Foundation Grant GP-1816. 
argument (see [1, Lemma 2.4]) that this last series is finite.

As a corollary to the theorem we note that

$$
\sum_{n=1}^{\infty}(-1)^{n} n^{-1} P\left(\left|S_{n}\right|>n \epsilon\right)
$$

is convergent for all $\epsilon>0$. In [2] it is shown that this series is absolutely convergent for all $\epsilon>0$ if and only if $E X_{1}=0$.

\section{REFERENCES}

1. M. Rosenblatt, On the oscillation of sums of random variables, Trans. Amer. Math. Soc. 72 (1952), 165-178.

2. F. Spitzer, A combinatorial lemma and its application to probability theory, Trans. Amer. Math. Soc. 82 (1956), 323-339.

UNIVERSITY OF MisSOURI AND

University of NeW MeXico

\section{THE BOUNDARY OF THE RANGE OF A VECTOR MEASURE}

VOLKER BAUMANN

Let $(X, S)$ be a measure space, $\mu_{i}(i=1, \cdots, n)$ signed measures on $(X, S)$. Then $\mu=\left(\mu_{1}, \cdots, \mu_{n}\right)$ is a (n-dimensional) vector measure on $(X, S), \mu$ is finite and purely nonatomic if every $\mu_{i}$ is finite and purely nonatomic, respectively. Consider the range of a finite $n$ dimensional vector measure as a subset of the $n$-dimensional Euclidean space $E^{n}$. A. Liapounoff [4] and P. R. Halmos [2] have shown:

(1) The range of a finite vector measure is closed,

(2) the range of a finite and purely nonatomic vector measure is convex.

For any (infinite) vector measure $\mu$ call $R=\{\mu(M): M \in S$ and $\mu(M)$ finite $\}$ the finite range of $\mu$. Then it is an immediate consequence of (2) that

(3) the finite range of a purely nonatomic vector measure is convex.

Two simple examples due to R. Borges [1] however show that there are purely nonatomic as well as purely atomic vector measures the finite range of which is not closed:

(a) $S$ is the $\sigma$-ring of the one-dimensional Lebesgue sets, $\mu_{1}$ the Lebesgue measure and $\mu_{2}(M)=\int_{M} \exp \left(-z^{2}\right) d z, M \in S$. The positive

Received by the editors January 2, 1964. 